J Neuroophthalmol. 2016 March ; 36(1): 92-97. doi:10.1097/WNO.0000000000000338.

\title{
Innocent until proven guilty
}

\author{
Heather E. Moss, MD, PhD ${ }^{1,2}$, Tiffani Stroup, $\mathrm{DO}^{3}$, Amy Y. Lin, $\mathrm{MD}^{1,4}$, Oliver Graf, MD ${ }^{4}$, \\ Aaron Halfpenny, DO ${ }^{4}$, Howard L. Lipton, MD ${ }^{2,5}$, Ari M. Blitz, MD ${ }^{6}$, and Tibor Valyi-Nagy, \\ $\mathrm{MD}, \mathrm{PhD}^{4}$ \\ ${ }^{1}$ Department of Ophthalmology and Visual Sciences, University of Illinois at Chicago, Chicago, IL \\ ${ }^{2}$ Department of Neurology and Rehabilitation, University of Illinois at Chicago, Chicago, IL \\ ${ }^{3}$ Department of Neurology, University of Chicago, Chicago, IL \\ ${ }^{4}$ Department of Pathology, University of Illinois at Chicago, Chicago, IL \\ ${ }^{5}$ Department of Microbiology and Immunology, University of Illinois at Chicago, Chicago, IL \\ ${ }^{6}$ Department of Radiology and Radiological Sciences, Johns Hopkins Medical Center, Baltimore, \\ MD
}

\section{Dr. Moss}

A 31 year-old man experienced right eye blurring and pain. Magnetic resonance imaging (MRI) of the orbits showed thickening and enhancement of the right optic nerve extending from the globe to just before the chiasm. Intravenous (IV) steroids were administered to treat optic neuritis but discontinued after two doses because of an adverse reaction.

Nine days later he developed headache and worsening vision, became confused and was readmitted to the hospital. Visual acuity was no light perception (NLP) with the right eye and 20/20 with the left eye with a nasal field deficit. There was bilateral optic disc elevation without hemorrhages or cotton wool spots. He had receptive aphasia and right-sided weakness. Computed tomography (CT) of the head revealed a left temporo-parietal hypodensity with mass effect thought to represent infarction. Two days later he developed low-grade fever, flaccid paraplegia and a T4 sensory level. Antibiotics were started for presumed infectious transverse myelitis. Three days later he became lethargic. Head CT showed petechial hemorrhage in the left hemispheric lesion. He was transferred to our institution for further care. We performed a repeat MRI of the orbits, brain and spine as well as MR spectroscopy and a four-vessel catheter angiogram.

\section{Dr. Blitz}

MRI of the orbits shows extensive enlargement and enhancement of the right optic nerve (Fig 1 upper left). MRI of the brain shows a left temporo-parietal lesion with patchy enhancement, focal diffusion restriction, mass effect and hemorrhage (Fig 1 right). MRI of

Corresponding Author: Heather E. Moss, MD, PhD, UIC Ophthalmology (MC 648), 1855 W Taylor St, Chicago, IL 60612, 312-996-9120v 312-413-7895f, hemoss@uic.edu.

Conflicts of Interest: none 
the spine shows multiple, non-enhancing, longitudinally extensive, cervical and principally mid-to-upper thoracic spinal cord lesions (Fig 1 lower left). Perfusion images (not shown) showed increased tissue transit time and decreased relative cerebral blood volume in the hemispheric lesion. MRI spectroscopy showed elevated lipid/lactate peak, increased choline peak and decreased $\mathrm{N}$-acetylasparate peak. Four-vessel catheter-based cerebral angiography revealed no vascular abnormalities. Overall, the findings are most suggestive of ischemic or necrotic lesions, perhaps representing an infectious or inflammatory encephalomyelitis.

\section{Dr. Moss}

Extensive serological testing was unrevealing (Table 1). He was treated with IV methylprednisolone and plasma exchange followed by an oral steroid taper for presumed hemorrhagic leukoencephalitis.[1] Visual acuity improved to count fingers (CF) with the right eye. Cerebrospinal fluid analysis 30 days after treatment revealed a lymphocytepredominant pleocytosis and elevated protein, but was unrevealing of a specific diagnosis (Table 1).

Two months after presentation the patient lost vision in the left eye to NLP. Right eye vision remained CF. Neurological exam was otherwise unchanged. Another MRI of the brain and spinal cord was performed.

\section{Dr. Blitz}

The previously seen right optic nerve enhancement has resolved but there is now diffuse enhancement of the left optic nerve (Fig 2 left) and there are new regions of enhancement of the mid-to-lower thoracic spinal cord (Fig 2 right), likely at least in part reflecting new enhancing lesions rather than development of enhancement solely in the regions of pathology previously seen. The hemispheric lesion persists and is unchanged in appearance.

The bilateral sequential optic nerve enhancement as well as multiple spinal cord lesions separated in time and space suggests an ongoing acute demyelinating disorder. Tumefactive demyelination can certainly produce mass effect, although the extent of mass effect in this case would be unusual. Likewise, hemorrhage is not a commonly encountered finding in the typical demyelinating disorders but may be encountered in rare forms such as acute hemorrhagic leukoencephalitis.

\section{Dr. Moss}

Further serum and CSF testing revealed improved but ongoing protein elevation in the CSF, but was otherwise unrevealing (Table 1). CT chest, abdomen and pelvis did not show any lesions. Scrotal ultrasound was normal. Bone marrow biopsy was unrevealing. Accordingly, it was elected to biopsy the spinal cord.

\section{Dr. Valyi-Nagy}

A spinal cord biopsy at T10 demonstrated small portions of leptomeninges and spinal cord parenchyma with chronic inflammation dominated by macrophages and extensive necrosis and loss of spinal cord parenchyma. Neither a neoplastic nor an infectious process was 
detected. Special histological stains, immunostains and electron microscopy (EM) for bacteria, viruses, fungi and mycobacteria were negative. The findings were consistent with a late subacute-to-early chronic stage of an ischemic process or a necrotizing demyelinating process such as neuromyelitis optica (NMO). The biopsy findings did not favor MS because of the significant necrosis and tissue loss.

\section{Dr. Moss}

In view of the biopsy findings, the patient was suspected of having NMO and was treated with IV methylprednisolone, 5 cycles of plasma exchange, and cyclophosphamide. Left eye vision subsequently recovered to 20/800; however, right eye vision remained CF.

Seven months after presentation, while on prednisone 80mg daily, the patient's vision declined to light perception with the right eye and hand motion with the left eye. He received rituximab with improvement in left eye vision to 20/400. Nine months after initial symptoms, while hospitalized with MRSA bacteremia, his mental status deteriorated and another MRI of the brain was performed.

\section{Dr. Blitz}

This MRI shows an extensive lesion involving the brainstem and cerebellum with mass effect, patchy enhancement, focal diffusion restriction (not shown), and hemorrhage (Fig 3). In addition, the corpus callosum demonstrates extensive FLAIR hyperintensity, and there is now mass effect on the region of the cerebral aqueduct with developing supra-tentorial hydrocephalus.

\section{Dr. Moss}

Shortly after undergoing the MRI, the patient lost brainstem reflexes and did not recover. Following organ donation, an autopsy was performed.

\section{Dr. Valyi-Nagy}

Examination of the brain revealed cerebral edema with uncal and cerebellar tonsillar herniation. There was a partially cavitated lesion $(8 \times 7 \times 4 \mathrm{~cm})$ with ill-defined borders deep in the left cerebral hemisphere involving the temporal, parietal, and occipital lobes. The brainstem and cerebellum were focally softened and distorted. Sectioning of the cerebellum and brain stem revealed an ill-defined, softened lesion extending from the medulla into the midbrain, right thalamus and right cerebellar hemisphere. The lower spinal cord demonstrated atrophy and discoloration starting in the lower thoracic region.

Histopathologic examination of the left parietal lesion showed predominantly chronic changes, including macrophagic infiltrates, gliosis and focal cavitation (Fig 4A). LFB-PAS and neurofilament stains demonstrated significant myelin and axon loss. GFAP staining highlighted extensive gliosis. Special stains for bacterial, fungal, and acid fast organisms (Gram, GMS, PAS, AFB) were negative. Immunostains for HSV-1, HSV-2, and polyomavirus were negative. 
Acute lesions were detected in the brainstem, cerebellum, and right basal ganglia with extensive, focally necrotizing inflammation (Fig 4B). Inflammatory infiltrates were primarily composed of neutrophils but a significant number of CD68-positive macrophages and a smaller number of CD3-positive T-cells were also noted. Only very rare CD20positive B-cells were detected. There was vascular and perivascular deposition of eosinophilic material. GFAP staining highlighted extensive fragmentation of astrocytic processes in perivascular areas (Fig 4C). Luxol fast blue-PAS special staining and neurofilament immunostaining indicated no selective loss of myelin (Fig 4D). There was a perivascular loss of aquaporin 4 (AQP4) immunoreactivity (Figs 4E and F). Immunostains for HSV-1, HSV-2, CMV, and polyomavirus were negative. Special stains for bacterial, fungal, and acid fast organisms (Gram, GMS, PAS, AFB) were also negative. EM showed no evidence of viral organisms. Immunofluorescence staining demonstrated perivascular reactivity for $\mathrm{C} 3, \mathrm{C} 4$, and $\mathrm{IgG}$ and no specific reactivity for IgM, C1q, Kappa and Lambda. Viral, bacterial and acid fast cultures taken from the cerebellum postmortem were negative.

Histopathologic examination of the spinal cord revealed predominantly chronic lesions, with parenchymal tissue loss associated with cavitation and macrophagic infiltrates. Specifically, sections from the lower spinal cord demonstrated atrophy and extensive multifocal cavitation associated with macrophagic infiltrates in spinal cord parenchyma (Fig 4G). Acute inflammatory infiltrates also were present in the leptomeninges. Immunohistochemical and special stains demonstrated significant myelin and axon loss in cord parenchyma and extensive gliosis. Special stains for bacterial, fungal, and acid fast organisms (Gram, GMS, PAS, AFB) were negative.

Sections of the left and right eyes and optic nerves demonstrated multifocal, predominantly chronic but focally acute inflammatory infiltrates in the subarachnoid space around atrophic optic nerves (Fig 4H). Neurofilament and GFAP immunostains and Masson trichrome special staining were indicative of optic nerve atrophy and gliosis. Gram and GMS special stains were negative for bacteria and fungi. There was marked loss of retinal ganglion cells in the retina bilaterally (Fig $4 \mathrm{I})$.

The pathologic findings, including perivascular deposition of $\operatorname{IgG}$, complement, neutrophilic and eosinophilic infiltrates, extensive tissue necrosis throughout the CNS, destruction of perivascular astrocytes, and loss of AQP4 support a pathological diagnosis of NMO.

\section{Final Diagnosis}

Neuromyelitis optica (NMO)

\section{Dr. Moss}

Clinical categorization of demyelinating diseases is challenging due to overlapping phenotypes and definitions dependent on clinical course. In our case, a disease on the demyelinating spectrum was the favored diagnosis from the outset, based on nearly concurrent optic neuritis, transverse myelitis and the hemispheric lesion. The prominent hemorrhagic hemispheric lesion dissuaded us from an initial diagnosis of NMO and led us to favor hemorrhagic leukoencephalitis, a monophasic neuro-inflammatory illness.[1] When 
the chronic relapsing nature of the patient's disease became evident, a clinical diagnosis of NMO was made. We attribute the hemispheric lesion to an atypical demyelinating lesion of the type that is increasingly recognized and do not think it to be incompatible with NMO.[2, 3] AQP4-IgG seronegative status, as was the case in our patient, does not rule out the diagnosis of NMO, as the sensitivity of commercially available tests is around 65\%.[4] It is not known if AQP-4-IgG was present in the CSF, as has been reported in some seronegative NMO patients.[5] Histopathological distinctions are increasingly helpful in distinguishing demyelinating diseases, as demonstrated in our case.[6] Ultimately, findings of perivascular loss of AQP4, perivascular fragmentation of astrocytic processes and lack of selective myelin loss on postmortem histopathology of the hyperacute cerebellar lesion confirmed the diagnosis of NMO.[2,7]

Our case provides insight into the mechanism of tissue injury in NMO, including the relative timing of astrocytic injury versus demyelination due to oligodendrocyte injury, which remains incompletely understood.[2, 8] Anti AQP4-IgG, a serum autoantibody targeting the water channel AQP4, binds at or near the blood-brain barrier and is an important feature of NMO pathogenesis. $[9,10]$ Astrocytic foot processes containing AQP4 are thought to be the primary targets of the immune system attack in NMO. Therefore, NMO has been proposed to represent a demyelinating disease characterized pathologically by demyelination that is secondary to acute destruction of perivascular astrocytes. Histopathological study of acute lesions supports this theory.[8, 11] Alternatively, it has been proposed that, in a subset of NMO cases, oligodendrocyte apoptosis and selective loss of minor myelin proteins occurs simultaneously with astrocyte pathology.[2] We present here a fatal case of NMO with a large hyperacute lesion demonstrating extensive astrocytic pathology without significant selective demyelination. These findings provide further support to the hypothesis that demyelination in NMO is a secondary event by demonstrating astrocyte injury predating demyelination in this pathologically proven case.

\title{
Acknowledgments
}

\author{
Funding: \\ K12 EY 021475 (HM) \\ K23 EY 024345 (HM) \\ Departmental Grant from Research to Prevent Blindness (HM, AL) \\ NIH R01 NS077755 (HL) \\ National Multiple Sclerosis Society (HL) \\ Modestus Bauer Foundation (HL)
}

\section{References}

1. Ryan LJ, Bowman R, Zantek ND, Sherr G, Maxwell R, Clark HB, Mair DC. Use of therapeutic plasma exchange in the management of acute hemorrhagic leukoencephalitis: a case report and review of the literature. Transfusion. 2007; 47:981-986. [PubMed: 17524086] 
2. Bruck W, Popescu B, Lucchinetti CF, Markovic-Plese S, Gold R, Thal DR, Metz I. Neuromyelitis optica lesions may inform multiple sclerosis heterogeneity debate. Ann Neurol. 2012; 72:385-394. [PubMed: 23034911]

3. Sato DK, Lana-Peixoto MA, Fujihara K, de Seze J. Clinical spectrum and treatment of neuromyelitis optica spectrum disorders: evolution and current status. Brain Pathol. 2013; 23:647660. [PubMed: 24118482]

4. Waters PJ, McKeon A, Leite MI, Rajasekharan S, Lennon VA, Villalobos A, Palace J, Mandrekar JN, Vincent A, Bar-Or A, Pittock SJ. Serologic diagnosis of NMO: a multicenter comparison of aquaporin-4-IgG assays. Neurology. 2012; 78:665-671. discussion 669. [PubMed: 22302543]

5. Klawiter EC, Alvarez E 3rd, Xu J, Paciorkowski AR, Zhu L, Parks BJ, Cross AH, Naismith RT. NMO-IgG detected in CSF in seronegative neuromyelitis optica. Neurology. 2009; 72:1101-1103. [PubMed: 19307546]

6. Popescu BF, Lucchinetti CF. Pathology of demyelinating diseases. Annu Rev Pathol. 2012; 7:185217. [PubMed: 22313379]

7. Popescu BF, Guo Y, Jentoft ME, Parisi JE, Lennon VA, Pittock SJ, Weinshenker BG, Wingerchuk DM, Giannini C, Metz I, Bruck W, Shuster EA, Carter J, Boyd CD, Clardy SL, Cohen BA, Lucchinetti CF. Diagnostic utility of aquaporin- 4 in the analysis of active demyelinating lesions. Neurology. 2015; 84:148-158. [PubMed: 25503621]

8. Parratt JD, Prineas JW. Neuromyelitis optica: a demyelinating disease characterized by acute destruction and regeneration of perivascular astrocytes. Mult Scler. 2010; 16:1156-1172. [PubMed: 20823059]

9. Verkman AS, Phuan PW, Asavapanumas N, Tradtrantip L. Biology of AQP4 and anti-AQP4 antibody: therapeutic implications for NMO. Brain Pathol. 2013; 23:684-695. [PubMed: 24118484]

10. Levy M, Wildemann B, Jarius S, Orellano B, Sasidharan S, Weber MS, Stuve O. Immunopathogenesis of neuromyelitis optica. Adv Immunol. 2014; 121:213-242. [PubMed: 24388217]

11. Barnett MH, Prineas JW, Buckland ME, Parratt JD, Pollard JD. Massive astrocyte destruction in neuromyelitis optica despite natalizumab therapy. Mult Scler. 2012; 18:108-112. [PubMed: 21868485] 

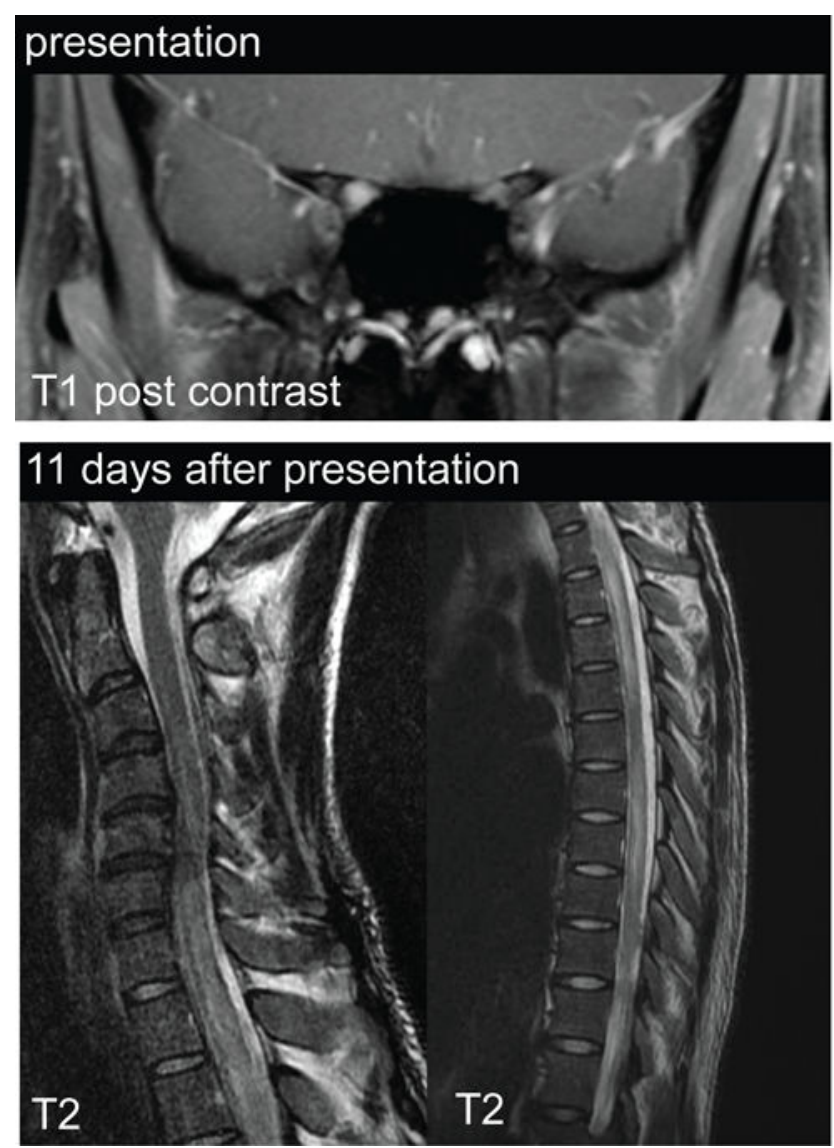

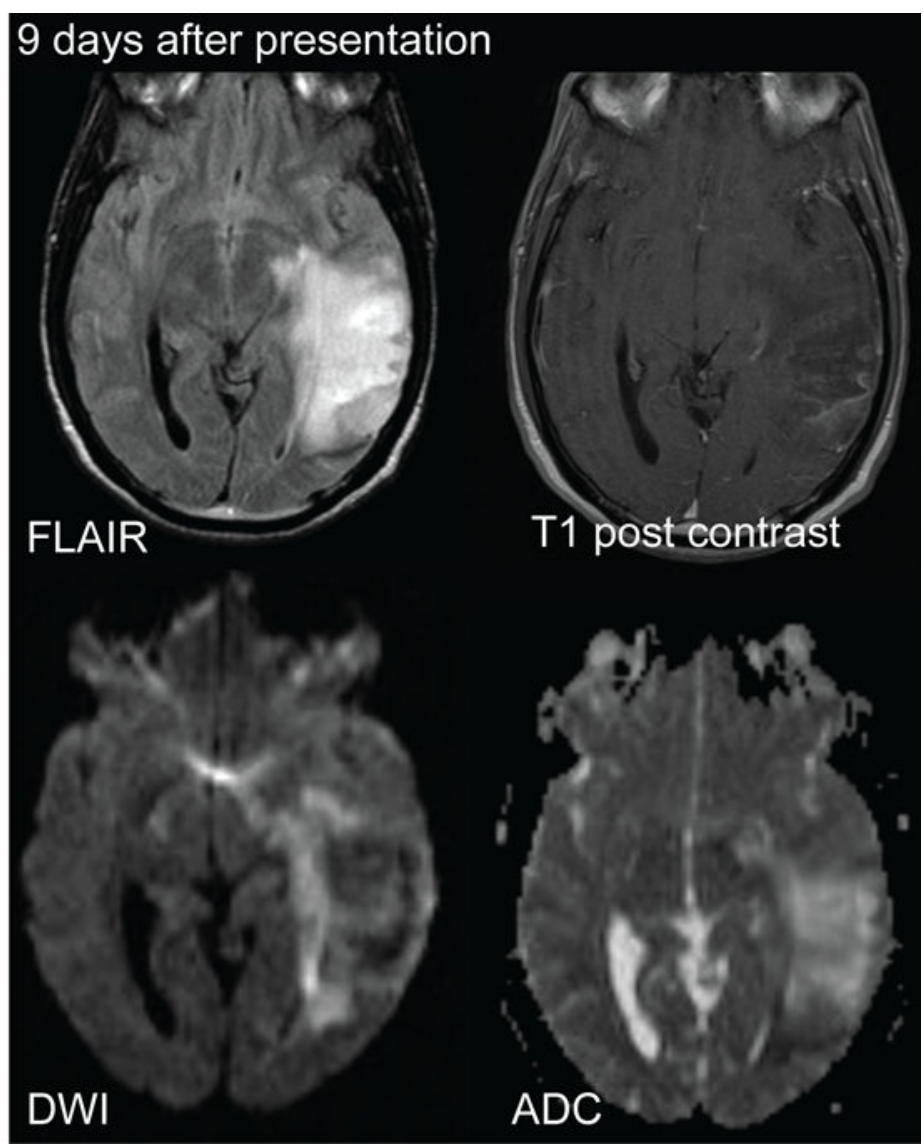

Figure 1.

coronal T1 post contrast MRI brain at time of presentation with right optic neuropathy demonstrationg right intracranial optic nerve enhancement (top left). Axial FLAIR, T1 post contrast, diffusion weighted and apparent diffusion coefficient MRI brain obtained after development of right hemiparesis ( 9 days after initial presentation) demonstrating a large left hemispheric lesion with edema, patchy enhancement, and areas of restricted diffusion (right panel). Sagittal T2 MRI cervical and thoracic spine MRI obtained after development of paraplegia, 11 days after initial presentation, showing multiple longitudinally extensive, expansive central cord lesions (bottom left). 


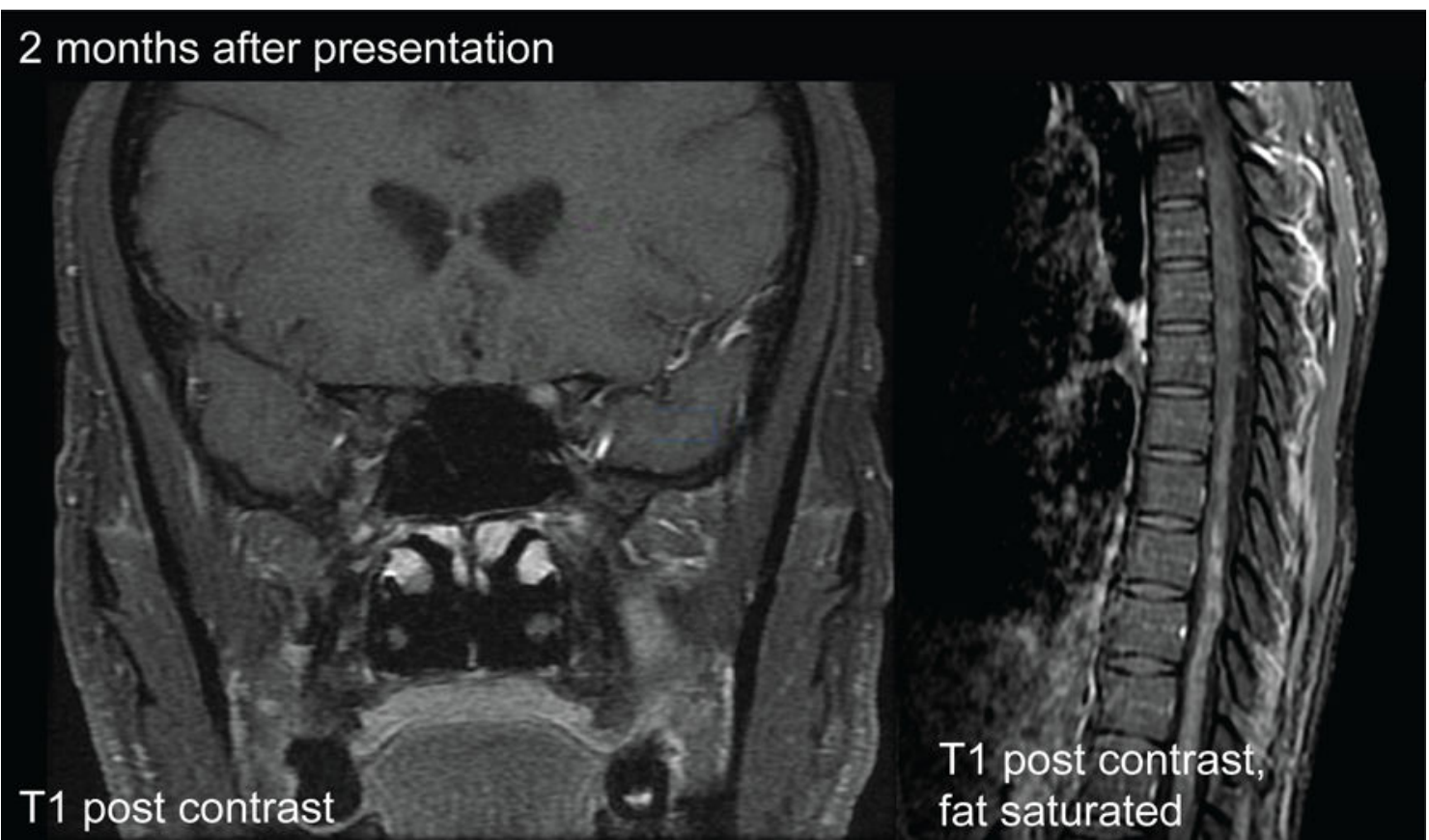

Figure 2.

Coronal T1 post contrast MRI brain (left) and sagittal T1 post contrast fat saturated MRI thoracic spine obtained at time of development of left optic neuropathy, 2 months after initial presentation, showing interval development of left intracranial optic nerve enhancement and new patchy enhancement of the spinal cord lesions. 


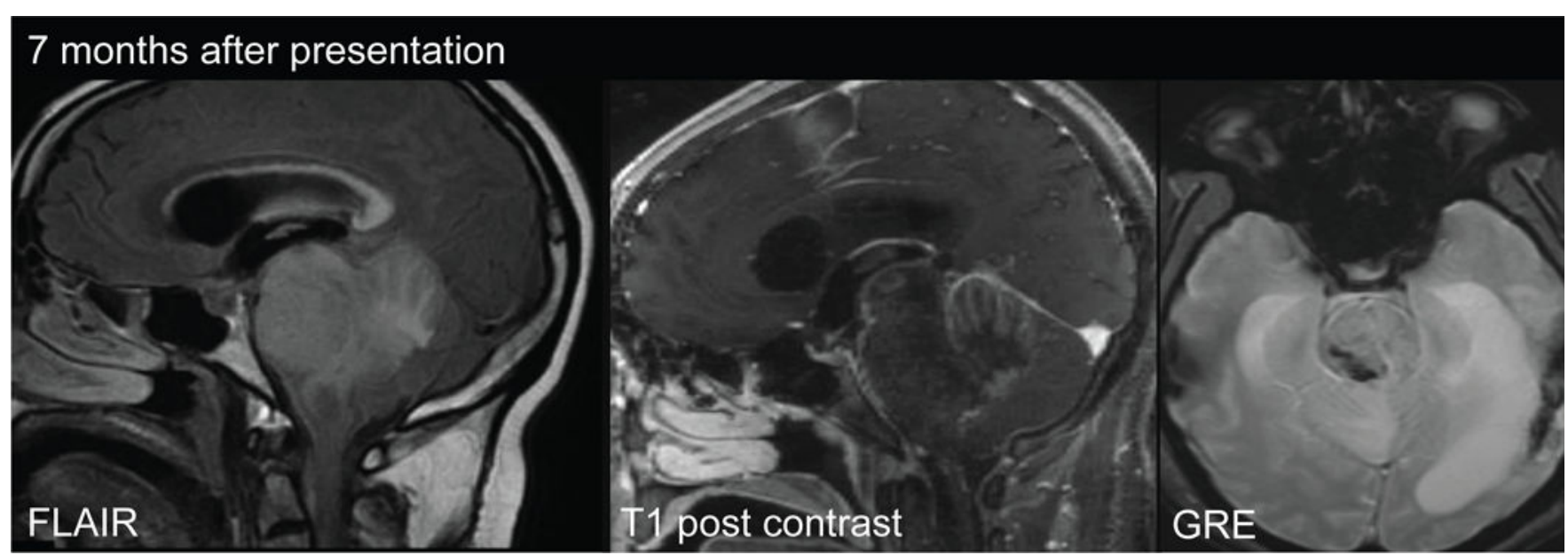

Figure 3.

Sagittal FLAIR (left), T1 post contrast (center) and axial gradient echo (right) MRI brain obtained following acute neurological deterioration, 7 months after initial presentation, demonstrating large brainstem and cerebellar lesions with edema, patchy enhancement and hemorrhage. 

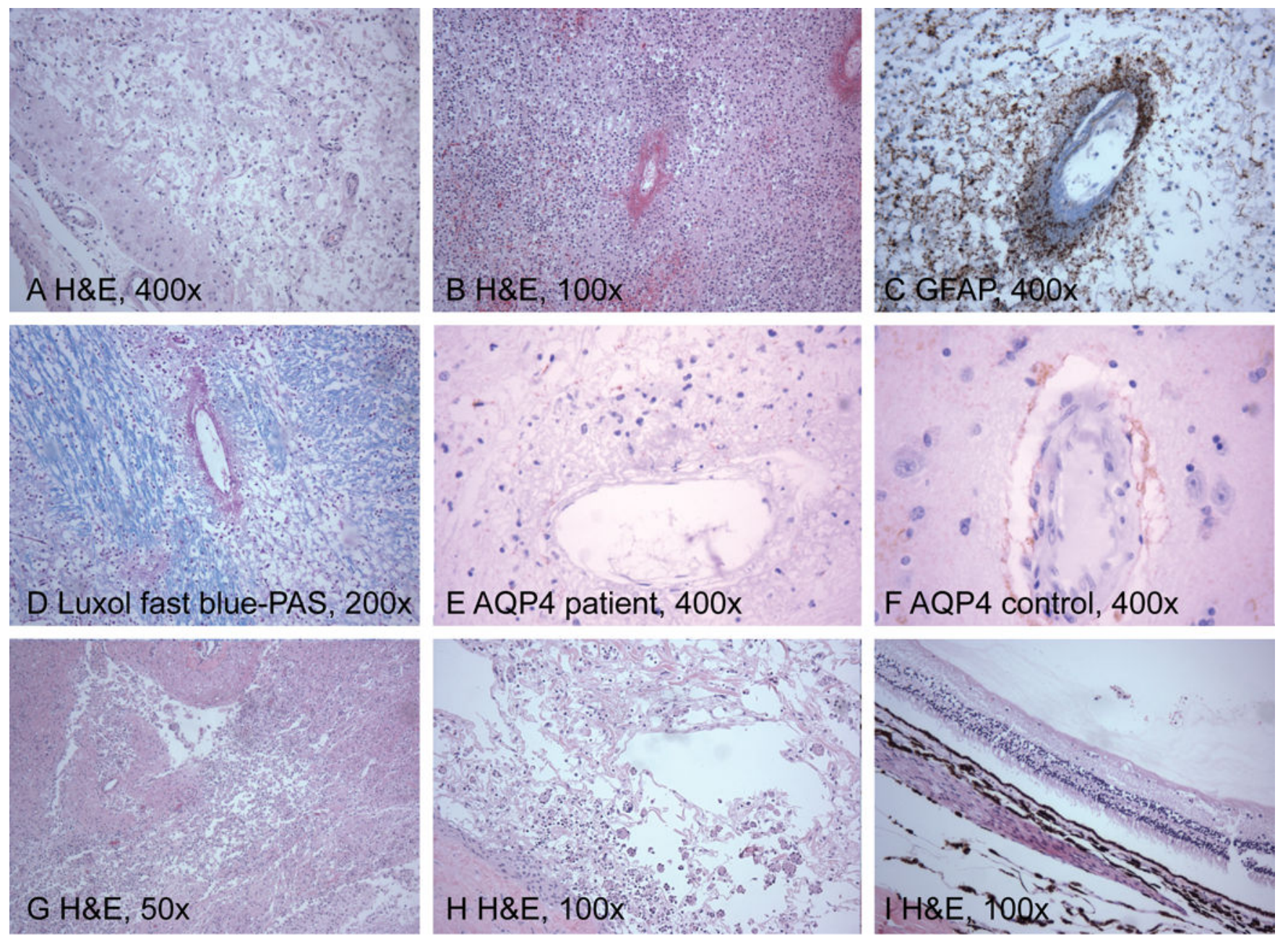

Figure 4.

Histo-pathology specimens obtained at autopsy, 7 months after initial presentation.

Hematoxylin and eosin stain of the parietal lesion demonstrating sequelae of chronic inflammation (A 50x). Hematoxylin and eosin (B, 100x), GFAP (C, 400x), Luxol fast bluePAS (D, 200x) and anti-aquaporin-4 (E, 400x) stains of the cerebellar lesion and aquaporin-4 control (F, 400x) demonstrating acute necrotizing inflammation, astrocytic fragmentation, perivascular loss of aquaporin-4 immunoreactivity and no loss of myelin. Hematoxylin and eosin stains of the chronic spinal cord lesion with chronic inflammation $(\mathrm{G}, 50 \times)$, optic nerve with mixed chronic and acute inflammation $(\mathrm{H}, 100 \times)$ and retina with marked loss of retinal ganglion cells(I, 100×) 


\section{Table 1}

Laboratory Testing (Bold denotes positive result)

\begin{tabular}{|c|c|}
\hline At time of right eye vision loss (0-1 months after presentation) & At time of left eye vision loss ( 2 months after presentation) \\
\hline \multicolumn{2}{|c|}{ Serum tests (inflammatory) } \\
\hline Anti-aquaporin $4 \mathrm{ab}$ & Anti-aquaporin $4 \mathrm{ab}$ \\
\hline Complement: $\mathrm{C} 3, \mathrm{C} 4, \mathrm{CH} 50$ & - \\
\hline ANA, anti DS-DNA & - \\
\hline Anti-RNP, anti-Smith & - \\
\hline $\mathrm{SSA} / \mathrm{B} \mathrm{ab}$ & - \\
\hline Lupus inhibitor & - \\
\hline Anti-cardiolipin ab & - \\
\hline Anti B2 glycoprotein & - \\
\hline- & SPEP IgG Kappa monoclonal gammopathy \\
\hline- & ANCA \\
\hline- & Cryoglobulins \\
\hline- & Beta-2 microglobulin \\
\hline \multicolumn{2}{|c|}{ Serum tests (infectious) } \\
\hline HSV I/II PCR & HSV I/II PCR \\
\hline EBV IgG +, IgM neg, ag neg & $\mathrm{EBV}$ ab \\
\hline CMV PCR & CMV ab \\
\hline Hepatitis B,C ab, ag & - \\
\hline HIV ab & - \\
\hline Coxsakie virus ab & - \\
\hline Quantiferon TB gold & - \\
\hline VZV PCR & - \\
\hline JCV PCR & - \\
\hline- & Lyme ab \\
\hline- & Bartonella Henselae IgG, IgM \\
\hline- & Bartonella Quintata IgG, IgM \\
\hline- & HTLV I/II IgG, IgM \\
\hline
\end{tabular}

J Neuroophthalmol. Author manuscript; available in PMC 2017 March 01. 


\begin{tabular}{|c|c|}
\hline At time of right eye vision loss (0-1 months after presentation) & At time of left eye vision loss ( 2 months after presentation) \\
\hline \multicolumn{2}{|c|}{ Cerebral spinal fluid } \\
\hline (30days post treatment) & On re-presentation \\
\hline $12 \mathrm{wbc}$ & $0 \mathrm{wbc}$ \\
\hline $0 \mathrm{rbc}$ & $0 \mathrm{rbc}$ \\
\hline Protein $140 \mathrm{mg} / \mathrm{dL}$ & Protein $76 \mathrm{mg} / \mathrm{dL}$ \\
\hline No oligoclonal bands & No oligoclonal bands \\
\hline myelin basic protein $140 \mathrm{ng} / \mathrm{dL}$ & myelin basic protein $279 \mathrm{ng} / \mathrm{mL}$ \\
\hline HSV I/II PCR & HSV I/II PCR \\
\hline EBV PCR & EBV PCR \\
\hline CMV PCR & CMV PCR \\
\hline VZV PCR & VZV PCR \\
\hline cryptococcal ag & cryptococcal ag \\
\hline angiotensin converting enzyme & - \\
\hline- & $\begin{array}{l}\mathrm{IgG} \text { index } 1.64 \\
\text { IgG synthesis rate } 28 \mathrm{mg} / \text { day }\end{array}$ \\
\hline- & OCB neg \\
\hline- & JCV PCR \\
\hline- & Lyme ab \\
\hline- & WNV IgG, IgM \\
\hline \multicolumn{2}{|c|}{ Other } \\
\hline Nasopharyngeal viral PCR & - \\
\hline Urine histoplasma ag & - \\
\hline- & Serum paraneoplastic panel \\
\hline
\end{tabular}

